\title{
Zeitschriftenübersicht - Revue Analytique
}

The Journal of Obstetrics and Gynaecology of the British Empire Vol. 59, Oktober 1952, Nr. 5.

Verhandlungen des 13. britischen geburtshilf lichen gynäkologischen Kongresses 8./10. Juli 1952 in Leeds.

Corpus-Ca des Uterus, von van Bouwdijk Bastiaanse, Amsterdam (10 Tabellen, 13 Figuren).

217 vaginale Operationen ohne Todesfall, 33 abdominelle mit einem Todesfall an Embolie. Von den unter 50 Jahre alten waren nach 10 Jahren noch alle gesund, von den Altern noch 56\%. Abnorme Geburtsarbeit des Uterus, von G. Bill, Dundee und C. W. Nixon, London (13 Figuren, 1 Tabelle).

Diese beiden Hauptreferate und die zahlreichen Diskussionsvoten behandeln alle vielfach umstrittenen Fragen und müssen im Bericht selbst nachgelesen werden.

Die Aufgabe des Pädíaters in der Geburtshílfe, von W. Gaisford, Manchester.

Die Besprechung der verschiedenen Möglichkeiten und Notwendigkeiten von Mitarbeit des Säuglingsarztes führt zum Hauptpostulat: Verminderung der Früh-geburten. An diesem Problem müssen aber nicht nur die Pädiater, sondern auch Pathologen, Physiologen und Politiker mitarbeiten. Die Frage wird in einer an-regenden Diskussion weiter erörtert.

Buchbesprechungen - Book Reviews - Lívres Nouveaux 387

Hartnäckíge Urinínkontínenz, von A. Aldrídge, New York (25 Figuren).

In seinem einführenden Referat empfiehlt der Autor auf Grund seiner eigenen Erfahrungen die nach ihm benannte Fascien-Scblingen-Operation, deren Ergebnis er bei 29 Operierten, mit nur zwei Mißerfolgen, bis zu acht Jahren nachkontrollierte. In der angeschlossenen Diskussion fanden auch die andern Verfahren ihre Vertreter.

Die weíblíche Genitaltuberkulose, von M. Haínes, London und J. Stallworthy, Oxford (4 Tabellen, 14 Figuren).

Während H. die Anatomie und Bakteriologie bespricht, hat S. den klinischen Teil des Referates übernommen. Eine sichere Diagnose kann meist vor der Opera tion oder ohne diese gestellt werden. Die Strahlentherapie ist heute noch umstritten. Streptomycin und PAS werden, nebst allgemeiner Kräftigung, empfohlen. Die Operation kann bei den heutigen medikamentösen Hilfsmitteln meist vermieden werden und bedarf sorgfältiger Auswahl und Vorbereitung. In der Diskussion wer den die Indikationen für die Strahlenbehandlung genau umschrieben und die giiristigen Resultate mit den heutigen medikamentösen Hilfsmitteln im Sinne des

Referates betont. Jung, St. Gallen/Meggen (LU)

Die medizinische Welt

1951, 20, Nr. 48

Die Wirkstoffe der allergíschen Reaktíon und ihre Bedeutungfür Diagnose und Therapíe, von G. Stüttgen (pg. 1503). 
Die Diagnostik und Therapie allergischer Vorgänge wird kurz behandelt. Haut-tests erweisen sich als wertvoll. Therapeutisch stehen Eliminierung des Antigens, Desallergisierung, Milieuwechsel, Antihistaminíca und Cortison im Vordergrund. «Variolabüe und Statotoniker», von W. Hellpach (p. 1510). Zwei konstitutionelle Extreme werden einander gegenübergestellt: Einerseits die Variolabilen, denen eine Wetteränderung zusetzt; andererseits die Statotoniker, die durch einen stationären Witterungszustand affiziert werden. Wettervariolabile fühlen sich auch im Leben am wohlsten in ruhigen, stabílen Verhältnissen; die Statotoniker hingegen werden durch die Veränderung als solche belebt und beschwingt. Ulrich Müller Buchbesprechungen - Book Reviews - Livres Nouveaux

GüntL·r, G. W.: Röntge@uroskopie. Nierenbecken und -kelche, Harnleiter, Blase, Harnröhre. G. Thieme, Stuttgart 1952. IV + 46 Seiten, 86 Abb., DM 13.50.

Verfasser empfiehlt die RU als Ergänzung zur Ausscheidungsurographie und an Stelle der «blinden» retrograden Urographie. Die Beobachtung der ableitenden Harnwege unter dem Durchleuchtungsschirm ermöglicht die Klärung diagnostiscli 